\title{
EMQN/CMGS best practice guidelines for the molecular genetic testing of Huntington disease
}

\author{
Monique Losekoot ${ }^{\star, 1}$, Martine J van Belzen ${ }^{1}$, Sara Seneca ${ }^{2}$, Peter Bauer ${ }^{3}$, Susan AR Stenhouse ${ }^{4}$ and \\ David E Barton ${ }^{5}$ on behalf of the European Molecular Genetic Quality Network (EMQN)
}

Huntington disease (HD) is caused by the expansion of an unstable polymorphic trinucleotide (CAG)n repeat in exon 1 of the HTT gene, which translates into an extended polyglutamine tract in the protein. Laboratory diagnosis of HD involves estimation of the number of CAG repeats. Molecular genetic testing for HD is offered in a wide range of laboratories both within and outside the European community. In order to measure the quality and raise the standard of molecular genetic testing in these laboratories, the European Molecular Genetics Quality Network has organized a yearly external quality assessment (EQA) scheme for molecular genetic testing of HD for over 10 years. EQA compares a laboratory's output with a fixed standard both for genotyping and reporting of the results to the referring physicians. In general, the standard of genotyping is very high but the clarity of interpretation and reporting of the test result varies more widely. This emphasizes the need for best practice guidelines for this disorder. We have therefore developed these best practice guidelines for genetic testing for HD to assist in testing and reporting of results. The analytical methods and the potential pitfalls of molecular genetic testing are highlighted and the implications of the different test outcomes for the consultand and his or her family members are discussed. European Journal of Human Genetics (2013) 21, 480-486; doi:10.1038/ejhg.2012.200; published online 19 September 2012

\section{DESCRIPTION OF THE DISEASE}

Huntington disease (HD, OMIM \#143100) is a progressive neurodegenerative disorder that presents with motor symptoms, cognitive impairment and psychiatric disturbances. The first symptoms usually manifest between 35 and 50 years of age and the duration of the disease is between 15 and 20 years. ${ }^{1,2}$ A small number of cases present before the age of 20 (juvenile onset) and about $25 \%$ of cases present after 50 years of age (OMIM). HD is inherited in an autosomal dominant fashion with an incidence of 3-10 in 100000 in populations of Western European descent. It is much less frequent (0.1-0.4: $100000)$ in other populations. ${ }^{1}$

$\mathrm{HD}$ is characterized pathologically by loss of specific neuronal populations in many brain regions, although the pathology is not limited to neurons. Neuropathological features include selective degeneration of neurons in the caudate and putamen and less severe loss in the cerebral cortex. More detailed information on the pathogenesis can be found in Ross and Tabrizi. ${ }^{3}$ Individuals homozygous for HD expansions appear to have a similar age of onset, but may exhibit an accelerated rate of disease progression. ${ }^{4}$

\section{THE GENE AND THE MUTATIONS}

The gene involved, the huntingtin (HTT) gene (NM_002111.6; NG_009378.1) previously known as IT15, is located on chromosome 4 p16.3, spans $180 \mathrm{~kb}$ and consists of 67 exons. The HTT gene is widely expressed and is required for normal development. It is expressed as two alternatively polyadenylated forms displaying different relative abundance in various fetal and adult tissues. The larger transcript is approximately $13.7 \mathrm{~kb}$ and is expressed predominantly in adult and fetal brain, whereas the smaller transcript of approximately $10.3 \mathrm{~kb}$ is more widely expressed. ${ }^{5}$

$\mathrm{HD}$ is caused by the expansion of an unstable polymorphic trinucleotide $(\mathrm{CAG}) \mathrm{n}$ repeat in exon 1 of the HTT gene, which translates into an extended polyglutamine tract in the protein. Alleles with $<27$ CAG repeats are classified as normal, whereas alleles with $\geq 36$ repeats are detected in patients (see Table 1 ). So far, the smallest number of CAG repeats described in patients with confirmed clinical features of $\mathrm{HD}$ is 36 . Alleles with 27-35 repeats (called mutable normal or intermediate alleles) are not associated with disease symptoms but can expand into the affected range upon (predominantly paternal) germline transmission and thus cause $\mathrm{HD}$ in offspring. Repeats of 36-39 CAG are incompletely penetrant and can be found in affected individuals as well as individuals who show no clinical symptoms in an advanced age ( $\geq 70-80$ years). The CAGrepeat number correlates inversely with the mean age of onset of symptoms. Generally, individuals with longer CAG repeats have an earlier age of onset. This is supported by findings that individuals with very large CAG repeats $(>60)$ present with juvenile $\mathrm{HD}$, and individuals with shorter CAG repeats (36-39) can remain asymptomatic. However, the number of repeats accounts only for approximately $70 \%$ of the variance in age at onset. ${ }^{6}$ This implies that not all juvenile cases have $\geq 60$ repeats. Numerous predicting models discussing the statistical relationship between the CAG-repeat length and the age of onset have been published over the last 15 years. A review and validation study of these statistical approaches can be found in Langbehn et al. ${ }^{7}$

${ }^{1}$ Department of Clinical Genetics, Laboratory for Diagnostic Genome Analysis, Leiden University Medical Centre, Leiden, The Netherlands; ${ }^{2}$ Centre for Medical Genetics, UZ Brussel and Reproduction and Genetics (REGE), Vrije Universiteit Brussel, Brussels, Belgium; ${ }^{3}$ Department of Medical Genetics, University Hospital of Tübingen, Tübingen, Germany; ${ }^{4}$ Duncan Guthrie Institute of Medical Genetics, Yorkhill Hospital, Glasgow, UK; ${ }^{5}$ National Centre for Medical Genetics, UCD School of Medicine and Medical Sciences, University College Dublin, Our Lady's Children's Hospital, Crumlin, Dublin, Ireland

*Correspondence: Dr M Losekoot, Department of Clinical Genetics, Laboratory for Diagnostic Genome Analysis, Leiden University Medical Centre, Leiden, The Netherlands. E-mail: m.losekoot@lumc.nl 
Table 1 Summary of the implications of different repeat ranges for the individual tested and family members

\begin{tabular}{|c|c|c|c|}
\hline \multirow[b]{2}{*}{ Number of repeats } & \multicolumn{2}{|c|}{ Implications for individual/patient } & \multirow[b]{2}{*}{ Implications for family members } \\
\hline & Diagnostic test & Predictive test & \\
\hline $6-26$ normal allele & Diagnosis not confirmed or diagnosis of HD excluded & Will not develop HD & No increased risk for HD \\
\hline 27-35 intermediate allele & Diagnosis not confirmed or diagnosis of HD excluded & Will not develop $H D^{b}$ & $\begin{array}{l}\text { Increased risk for HD } \\
(\text { few } \% ;<10 \%)^{c}\end{array}$ \\
\hline 36-39 incomplete penetrance allele & Diagnosis of HD confirmed & $\begin{array}{l}\text { May or may not develop HD; } \\
\text { in range of reduced penetrance }\end{array}$ & Increased risk for $\mathrm{HD}$ \\
\hline 40 and over complete penetrance allele & Diagnosis of HD confirmed & Will develop HD & Increased risk for HD \\
\hline
\end{tabular}

${ }^{\mathrm{a} A t}$ the higher end of the range one should use: 'diagnosis of HD is very unlikely'.

${ }^{\circ}$ At the higher end of the range one should use: 'it is very unlikely that the consultand will develop HD'.

${ }^{C}$ The figure depends on the type of intermediate allele $(I A)$. General population IAs have a much lower $(<1 \%)$ risk than IA that have already shown an expansion in the family.

Huntingtin is a protein of 3144 amino acids with a predicted molecular mass of $348 \mathrm{kD}$. The polyglutamine tract starts at residue 18 (c.52 p.18) and, when abnormally expanded, is thought to acquire a novel deleterious function. This eventually leads to neuronal dysfunction and neurodegeneration. The polyglutamine expansions also result in the formation of neuronal intranuclear inclusions containing huntingtin and ubiquitin.

\section{REPEAT SIZE RANGES}

\section{Normal alleles}

The CAG repeat is highly polymorphic in the population. Alleles in the range of 6-26 CAG have never been found to be associated with HD and there has been only one single report about a normal allele that was unstable on transmission to the next generation. ${ }^{8}$

\section{Disease size range}

The smallest number of CAG repeats described in patients with confirmed clinical features of $\mathrm{HD}$ is $36 .^{9,10}$ This repeat size has been observed in more than six documented HD cases. Although it is possible that HD cases with fewer repeats exist, only few $(n=5)$ possibly affected cases with $29-34$ repeats have been reported. ${ }^{11-14}$ Such cases are difficult to ascertain due to the paucity of alleles of this size. Although we cannot exclude the possibility that HD may be associated with these smaller repeat sizes, the published data are not conclusive and alternative diagnoses need to be carefully considered in such cases with HD-like signs.

\section{Range of reduced penetrance}

There have been many reports with elderly asymptomatic individuals with HD alleles of 36-39 repeats which define the range of incomplete or reduced penetrance of the mutation. ${ }^{9,10,15,16}$ Many carriers of these alleles remain asymptomatic until a very late age, although exceptions exist.

\section{Intermediate or mutable normal alleles}

Inconsistencies and confusion exist in literature regarding intermediate alleles despite the existence of published guidelines ${ }^{17}$ (personal observations during external quality assessment (EQA) for European Molecular Genetics Quality Network (EMQN)). These alleles of 27-35 repeats have been designated 'intermediate' and are also referred to as 'mutable normal' or 'large normal' alleles. They are defined as 'being below the affected range but having the potential to expand into the disease range in the next generation'. The lower limit is defined as the shortest size ever reported to expand into the HD range after one transmission. Intermediate alleles are relatively common in the general population with frequency estimates between 1 and $7 \% .^{17,18}$

The risk of expansion of an intermediate allele into the disease range has been estimated at $0.1-1 \%$ per generation; ${ }^{18,19}$ (see also next paragraph). Individuals who are found to carry alleles in this range should be counseled about the possibility of prenatal diagnosis and the risk for other family members. The small risks are difficult to quantify but may be more significant for males transmitting alleles in this range. Where intermediate and pathogenic alleles are found in the same individual, the interpretation should clearly distinguish the risks associated with each allele.

\section{REPEAT INSTABILITY}

Although the rate of new mutations in HD is low, these have been described and originate from intermediate alleles of 27-35 repeats, mainly through paternal transmission. ${ }^{20,21} \mathrm{Up}$ to now, only one maternal case has been documented. ${ }^{22}$ Factors influencing repeat instability include size of the allele, sex and age of the transmitting parent, family history and gene sequence and haplotype on which the intermediate allele is located. Alleles with a larger number of CAG repeats have a higher likelihood of expanding into the affected range upon transmission to the next generation. ${ }^{17}$ The risk that offspring will develop HD is the highest for fathers carrying these alleles who are $>35$ years of age. ${ }^{23}$ Intermediate alleles can be coincidently identified when healthy family members in an HD family undergo CAG-repeat sizing ('general population intermediate alleles'). In contrast, they can also be ascertained from new mutation families and these new mutation alleles are more prone to repeat expansion when compared with similar-sized alleles in the general population. The genetic variability near the repeat tract can influence its stability. The c.7934_7936del p.Glu2645del (known as the $\Delta 2642$ ) polymorphism is vastly overrepresented on HD chromosomes (38\%) relative to the general population (7\%). Finally, repeat tracts in which the 3 -CAA repeat has changed to a CAG are markedly more unstable (see Figure 1a). Both polymorphisms tend to cluster in specific haplotypes. ${ }^{24}$ Additional information is needed from various populations to determine a more precise and critical risk assessment for offspring of intermediate allele carriers.

\section{Anticipation}

Anticipation is the phenomenon in which increasing disease severity and/or decreasing age of onset is observed in successive generations. In HD, it occurs more commonly through paternal transmission. The phenomenon arises because of the expansion of the unstable CAG repeat during spermatogenesis. Also large expansions, for 


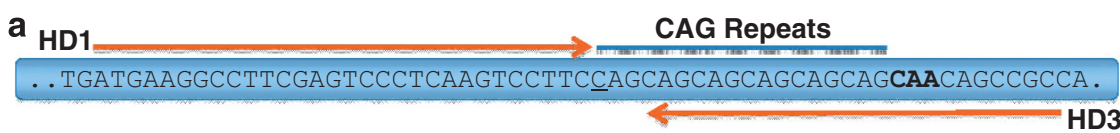

b

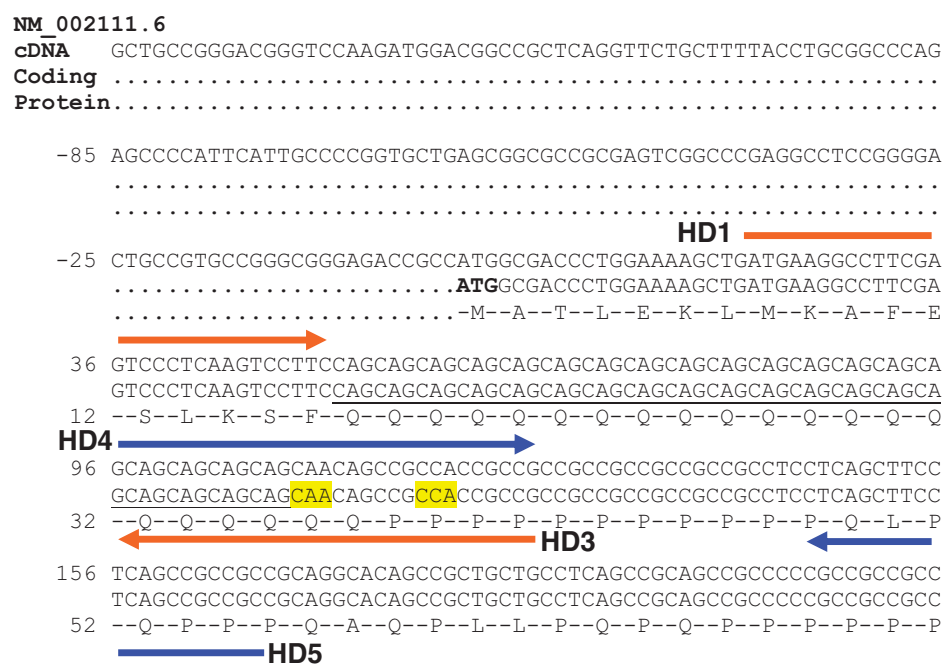

Figure 1 (a) The sequence immediately flanking the CAG repeat in the HTT gene. The sequence illustrated contains six CAG repeats. The position of the start of the CAG repeats at c.52 is underlined. The $3^{\prime}-$-CAA (shown in bold) and following CAG (both of which code for glutamine) are not counted. The positions of primers HD1 and HD3 from the commonly used assay of Warner et al. ${ }^{27}$ are indicated with orange arrows. The reverse primer, HD3, overlaps the last 13 bases of the CAG repeats. (b) The DNA and amino-acid sequence around the HTT exon 1 polyglutamine repeat. The first C of the CAG repeats is c.52 in CDNA sequence NM_002111.6. The start of the coding sequence is indicated in bold. This sequence contains 19 CAG repeats (underlined). The polymorphic CCG/polyproline sequence $3^{\prime}$ of the CAG repeats is shown and is not counted. The two mutated sequences found in patients with allelic drop out are given in yellow. The locations of some commonly used primers are indicated with arrows; HD1 and HD3 are indicated in orange. HD4 and HD5, which amplify the CCG repeat, are indicated in blue. HD1 and HD5 (or the originally published HD2, not shown) can be used together to amplify the CAGCCG repeat.

example, an allele size increment of $>7$ repeats, occur almost exclusively through paternal transmission and may result in juvenile onset of HD in the next generation. The chance of transmitting a large expansion also depends on the size of the parental CAG repeat: a carrier of a large expansion will have a higher risk of transmitting an even larger expansion to the offspring.

\section{REASONS FOR REFERRAL}

Confirmation of a clinical diagnosis

This is usually requested by a neurologist or a clinical geneticist. Depending on local policies, other treating physicians might request a confirmation or exclusion of a clinical diagnosis of HD. If the diagnosis is confirmed by DNA analysis, the patient and family members should be referred for genetic counseling and a possible offer of presymptomatic testing. Prenatal and preimplantation genetic diagnosis can be offered to at-risk couples. Although presymptomatic testing is usually not offered to them, minors with symptoms of juvenile $\mathrm{HD}$ can have diagnostic testing. If the diagnosis is confirmed, this will result in a presymptomatic bystander result for one of the parents if they are unaffected at the time. A specific presymptomatic test for the parent should therefore be considered in parallel, and an appropriate supporting structure for the family should be available.

\section{Presymptomatic testing}

According to the international guidelines, predictive testing for HD should only be requested by a clinical geneticist. ${ }^{25,26}$ Presymptomatic testing can be offered to individuals at an a priori 50 or $25 \%$ risk. On very rare occasions, an individual at $12.5 \%$ risk may be tested. Confirmation of $\mathrm{HD}$ at the molecular level in at least one family member with HD is advisable. Presymptomatic testing of minors $(<18$ years of age $)$ is considered appropriate only in exceptional circumstances. ${ }^{25,26}$ Comprehensive genetic counseling is required, and informed consent must be provided in accordance with local practices.

Prenatal testing and preimplantation genetic diagnosis Prenatal testing should be requested by a clinical geneticist. The testing method is identical to presymptomatic testing with some exceptions (see section on analytical methods). Preimplantation genetic diagnosis (PGD) may be a valuable option for couples who wish to avoid transmitting the disease without revealing their own status or to avoid termination of a pregnancy. It is only offered in a limited number of specialized centers and will not be discussed here (see also Rij van et $a l^{27}$ ).

\section{The exclusion test}

In cases where an individual with a $25 \%$ risk of developing HD requests predictive testing but the parent, who has a $50 \%$ risk, does not want to be informed about his or her risk, an exclusion test can be offered. This test is nowadays almost exclusively used in prenatal testing where the fetus is at $25 \%$ risk and the future parent at $50 \%$ risk. It is performed with linked polymorphic markers (see also interpretation section). Exclusion testing may not be available in all countries.

\section{ANALYTICAL METHODS}

The number of repeats is established by PCR analysis of the region encompassing the CAG repeat (Figure 1a), followed by fragment 
sizing through capillary or gel electrophoresis at sufficient resolution to allow separation of alleles with one repeat difference. ${ }^{28,29}$ Other methods with comparable resolution can also be applied. Regardless of the PCR-based strategy selected, it is important that the assay conditions are optimized to ensure the accurate and unambiguous determination of the number of repeats. Laboratories must validate the test by measuring allele sizes and calculate uncertainty for their tests. Genotyping results from the yearly EQA scheme for molecular genetic testing of HD from the EMQN show that over the last 3 years (2008-2010), 3-9\% of alleles fall outside the error limits set by the EMQN. For 2010, 51 out of 570 alleles were typed outside the error limits (which, at the time, were set at \pm 1 for alleles $<40$ repeats, and \pm 3 repeats for alleles $>39$ CAG repeats). In several cases, this was because of the fact that CAGCCG repeats are reported instead of CAG repeats. Two other studies reported similar results. ${ }^{30,31}$

\section{Controls}

PCR products containing CAG repeats migrate anomalously in electrophoresis, making conventional size ladders unreliable. Instead, control samples with well-defined repeat sizes at the borders of normal, intermediate, reduced penetrance and expanded repeats, as well as a large expansion (>60 repeats), should be used for allele sizing. The number of repeats in these controls should preferably be determined by DNA sequencing and/or by calibration against a certified reference material (see below).

A prenatal test is technically identical to a presymptomatic test but the maternal or both parental samples should be analyzed in the same run. If the fetal genotype is identical to the maternal genotype, maternal contamination should be excluded using polymorphic microsatellites or equivalent.

\section{Reference materials}

Certified reference materials are essential aids to the accurate and traceable calibration of measurement systems. The only certified reference material available for HD testing is SRM 2393 from the US National Institute of Standards and Technology. This panel of six genomic DNAs contains alleles ranging from 15 to 75 CAG repeats characterized by Sanger sequencing. A useful panel of well-characterized DNAs is available from the Coriell CDC Repository. ${ }^{32}$ A cell line has been established from a patient shown by sequencing to carry 24 and 35 repeats. This cell line (or DNA prepared from it) is available from the ECACC (http://www.ecacc.org.uk/). It is cell line number CM0034, ECACC ref. no: 95090133.

\section{Sizing accuracy}

It is the laboratory's responsibility to empirically determine the error limits (precision) of their assay. Acceptable error limits are \pm 1 repeat for alleles $\leq 42$ and \pm 3 repeats for alleles $>42$. The CAG repeat in the HTT gene is adjacent to a $3^{\prime}$-positioned CCG repeat, which is also polymorphic in length (Figure 1b). ${ }^{6,29}$ The original primer sets, used to size the CAG repeats, also included this CCG polymorphism and can thus result in misclassification of alleles. ${ }^{33}$ Therefore, this assay should not be used for routine sizing of the CAG repeats, but may be very helpful to resolve two homozygous normal $\mathrm{HD}$ alleles with identical numbers of CAG repeats but different numbers of CCG repeats.

Annual participation in an EQA scheme is not only necessary to allow the comparison of allele sizing with other laboratories but also for quality assurance, continuous validation, evaluation of reporting and continuous education. HD testing should only be performed in laboratories that are accredited according to ISO 15189 or equivalent. $^{34}$

To exclude the presence of a very large expansion that would be missed by PCR in a case with homozygosity of a normal CAG-repeat allele, the use of a southern blotting protocol (PstI-digested DNA hybridized with probe 4G6P1.7) or TP-PCR is recommended (also see the next paragraph). ${ }^{28}$ PCR of large $(>100)$ CAG-repeat tracks in DNA from fresh or frozen tissue samples is feasible. ${ }^{35}$ Because individuals with $>60$ repeats will present with a juvenile or earlyonset form of HD, it will be unlikely that adult onset cases are missed by PCR analysis alone.

For homozygous (normal) CAG-testing results, several approaches may be taken:

1. As mentioned above, the CAGCCG repeat should be genotyped, which might demonstrate the presence of two normal alleles (with identical CAG-repeat counts but heterozygosity for the CCG repeat)

2. Samples homozygous for CAG and CCG repeats should be analyzed alongside an appropriate large CAG-repeat positive control (demonstrating the sensitivity of the test for larger CAG expansions)

3. In a symptomatic test setting, the age at onset of symptoms should be available and discussed as to whether young onset HD can be excluded clinically; that is, if the patient has onset in his/her 40 or 50s, a very large expansion is most unlikely.

4. In a presymptomatic test setting, although the proband's age can be taken into account, it may be advisable to offer genotyping for the proband's parents in order to confirm homozygosity or to check allele segregation by STR analysis.

5. In the juvenile HD setting, an apparent homozygous (normal) CAG result has to be treated with caution unless segregation analysis with polymorphic markers has indicated the presence of two normal alleles. If parental samples are not available, southern blotting or TP-PCR should be considered. ${ }^{36}$

Three very rare polymorphisms have been described in the $3^{\prime}$ or $5^{\prime}$ primer used in some CAG-specific assays. ${ }^{37,38}$ These can potentially disrupt primer binding to an HD chromosome and result in the failure to amplify a pathogenic allele. ${ }^{37}$ Table 2 lists the factors that can influence the analytical specificity and sensitivity of the CAGsizing assay. Methods of mitigating false-positive and false-negative results are detailed. Finally, in some countries, it is common practice for presymptomatic tests to ask for a second independent blood sample to confirm the result. In general, measures to avoid sample swaps should be implemented in every laboratory offering a diagnostic service.

\section{CLINICAL SENSITIVITY AND SPECIFICITY}

The absence of HD pathology in an individual with $\geq 40$ repeats who died after living up to or past normal life expectancy has never been described. Therefore, a result of $\geq 40$ repeats is a $100 \%$ diagnostic of HD. ${ }^{44}$ CAG-repeat expansions account for $>99 \%$ of cases of HD and, therefore, the test with a result of $\geq 40$ repeats and all appropriate controls is $>99 \%$ sensitive. $^{44}$

\section{INTERPRETATION OF THE RESULTS AND REPORTING}

The interpretation should always be done in the context of the clinical referral and is summarized in Table 1 . It is important to make a clear distinction between a report for a diagnostic test on a patient with symptoms of $\mathrm{HD}$ and a report for presymptomatic testing. One has 
Table 2 Factors affecting diagnostic parameters in HD testing

\begin{tabular}{|c|c|}
\hline Parameter & Possible risks (pitfalls) \\
\hline $\begin{array}{l}\text { Analytical sensitivity } \\
\text { Sensitivity: The proportion of biological } \\
\text { samples that are rightfully classified as } \\
\text { positive by the test ( }=1 \text { - proportion of }\end{array}$ & $\begin{array}{l}\text { As a sensitive HD test is designed to } \\
\text { identify CAG-repeat expansions of } \\
\text { unknown size, there is a risk of missing } \\
\text { true positives (allelic drop out (ADO)). }\end{array}$ \\
\hline
\end{tabular}

Technical requirements

false-negative results)

- If the PCR test reveals two normal alleles in the sample, a false-negative result can be ruled out.

- The PCR assay must have the capacity to detect alleles in the normal and expanded ranges (up to 100 repeats), and resolve alleles one repeat apart

- Include positive control (>60 repeats) in every experiment (or allelic ladder)

- Primer design is cruciala

- Testing with primers spanning the CAGCCG repeat will help to distinguish two normal alleles in most cases

- TP-PCR or southern blotting will exclude or confirm the presence of a large expansion

- Exceptionally, an answer can be sought with segregation analysis with linked STR markers in extended pedigrees

- Only a few observations of polymorphisms at primer-binding sites are reported ${ }^{b}$

- Caution with automated allele detection tools: expansion-related stutters can result in small peak heights. Ensure the electropherogram extends far enough to visualize large expansions.

Analytical specificity Specificity: The proportion of biological samples that is rightfully classified as negative by the test ( $=1$-proportion of false-positive results)
A pathological CAG expansion in the HTT gene is the only biological reason for $\mathrm{HD}$
- Electropherograms should be monitored thoroughly for the presence of triplet stutter peaks in order not to mistake electrophoresis artifacts for HD alleles.

- The signals of PCR-amplified HD mutations in capillary electrophoresis represent a highly specific pattern, which is distinct from any other technical PCR or electrophoresis artifacts.

- Standard negative controls (blank without DNA template) are sufficient

apublished primers: see refs $33,39,40$

bee refs $20,37,38,41-43$

to bear in mind that the result is not only important for the consultand tested but is also relevant for the family members. Each laboratory has its own reporting format, but general reporting guidelines can be found on the EMQN website www.emqn.org linked to the CMGS guidelines for reporting (http://www.cmgs.org/BPGs/ Best_Practice_Guidelines.htm). One should also adhere to the ISO 15189 and OECD guidelines. ${ }^{34}$ A one-page report is the preferred format in which the test result and the answer to the clinical question should be easy to find and unambiguously formulated. The report is a stand-alone document that should not only be clear to the referring physician but also to other professionals involved in supporting and/ or treating the patient. The reports should carry a clear key including a short clinical interpretation of the different size ranges. Alleles at the boundaries of size ranges should be determined precisely by the use of appropriate reference materials and/or by use of the most precise method available; if the resultant genotype still includes more than one range (eg, $35 \pm 1$ CAG repeats), this should be reflected in the interpretation. The answer to the clinical question, the take-homemessage, should be stated clearly and unambiguously: for example, 'The diagnosis of HD either is or is not confirmed.' 'The consultand will or will not develop HD'. Although local policy can vary with regard to reporting, some relevant HD-specific items are mentioned below.

\section{Nomenclature}

The number of CAG repeats is measured as the number of uninterrupted CAG repeats (see Figures $1 \mathrm{a}$ and $\mathrm{b}$ ). There are more glutamines than CAG repeats in this part of the gene, as the polyglutamine tract is coded for in most chromosomes by $(\mathrm{CAG})_{\mathrm{n}}$ CAACAG (CAA also codes for a glutamine). The HGVS nomenclature is not considered the most appropriate for reporting the results of trinucleotide repeat analysis. Instead, the number of uninterrupted CAG repeats is used as a result of the genetic test. The error limits of the test should always be mentioned. Reporting actual allele sizes is subject to local practice. A statement whether the allele is in the normal or the disease range can very well be sufficient. However, it is important to report actual allele sizes if this is relevant for the interpretation of the result; for instance, in the case of a reduced penetrance or intermediate allele, as well as in the upper range of expansions where onset might be at a juvenile or infantile age.

\section{Diagnostic testing}

In the case of a CAG repeat $\geq 36$ the diagnosis of $\mathrm{HD}$ is confirmed (or is consistent with a diagnosis of HD). In the case of a CAG repeat $\leq 35$, the diagnosis is excluded (or the result is not consistent with HD). However, recently a few possibly affected cases with 29-34 repeats have been reported. ${ }^{11-14}$ Alleles in the reduced penetrance or intermediate (normal mutable) range have implications for family members, which should be discussed in the report. In these cases and in the case of a confirmation of HD, the family should be offered a referral for genetic counseling. In the case of a test result with two alleles in the normal range when disease symptoms are clearly present, the referring physician could be advised to consider reevaluating the clinical diagnosis and testing for HD-like diseases, such as HDL1 (PRNP gene), HDL2 (JPH3 gene), DRPLA (ATN1 gene) or SCA17. This may be mentioned in the report in more general terms.

\section{Predictive (presymptomatic) testing}

In cases of a CAG repeat from 36 to 39 , the individual is at risk of developing HD; in cases with a CAG repeat $\geq 40$, the individual will develop HD (or is at risk of developing HD). In cases of a CAG repeat $\leq 35$, the individual will not develop HD (or is not at risk of 
developing HD). Implications of alleles in the reduced penetrance or intermediate (normal mutable) range should be discussed in the report. Where available and appropriate, prenatal testing and PGD should be offered. Data in which the likelihood that an individual with a particular size CAG repeat will be affected by a specific age have been published. ${ }^{7,16,45}$ Although more accurate predictions can now be made, and these models are being used for recruitment for clinical trials, the $95 \%$ confidence intervals are still wide and they have not been validated for genetic counseling. Thus, extreme care should be taken applying this information in individual presymptomatic cases. In some cases of presymptomatic testing, the familial diagnosis is not confirmed by genetic testing. In such cases, an important caveat must be mentioned: a negative result excludes HD, but it does not necessarily exclude the familial disorder, which might be misdiagnosed. This must always be emphasized in the laboratory report.

\section{Prenatal testing}

The interpretation and approach to reporting a prenatal test is essentially the same as a presymptomatic test. However, it should be immediately clear from the report that it concerns a prenatal test. In addition, if the genotype of the fetus is identical to the maternal genotype, maternal contamination must be excluded and the result reported.

\section{Exclusion test}

If a person with an affected grandparent wants to know whether he/ she has an increased risk of developing HD, but their linking parent does not want to know his or her status, an exclusion test can be proposed. Nowadays, it is almost exclusively used as a prenatal test. The test result reveals whether the fetus (individual) with an a priori $25 \%$ risk received the grandparental risk haplotype or not (see also reason for referral). If the grandparental risk haplotype is present, the risk for the fetus is increased to approximately $50 \%$, without changing the risk for the $50 \%$ at risk parent. If the grandparental risk haplotype is not present, the fetus' risk is reduced to almost $0 \%$ (the recombination frequency between the markers and the gene should be taken into consideration). It is necessary to include DNA of at least one grandparent in the analysis. Confirmation of the diagnosis at the molecular level in at least one family member, preferably the grandparent with $\mathrm{HD}$, is necessary. For the exclusion test, polymorphic markers in the HTT region on chromosome $4 \mathrm{p} 16.3$ can be used (see Table 3 for a list of STRs). Ideally, markers both proximal and distal to HTT need to be informative to have a secure result. It is

Table 3 Suggested markers in $4 \mathrm{p} 16.3$ that are suitable for exclusion testing

\begin{tabular}{lrrrrr}
\hline Marker & UniSTS & $\begin{array}{c}\text { Ensembl } \\
\text { marker }\end{array}$ & $\begin{array}{c}\text { Ensembl } \\
\text { location (hg19) }\end{array}$ & $\begin{array}{c}\text { Het } \\
\text { frq }\end{array}$ & $\begin{array}{r}\text { Alias } \\
\text { (Ref) }\end{array}$ \\
\hline D4S2936 & 24920 & Z52740 & $692247-692420$ & & \\
D4S3038 & 42100 & Z51777 & $1099931-1100155$ & & \\
D4S1614 & 27925 & Z24429 & $2646689-2646866$ & & \\
D4S43 & 147240 & D4S43 & $2336363-2336628$ & 0.7 & C3946 \\
D4S127 & 149984 & D4S127 & $3038714-3038864$ & 0.7 & P36347 \\
HTT & & & $3076408-3245687$ & & \\
D4S3034 & 38369 & Z51717 & $3325536-3325722$ & 0.6 & \\
D4S412 & 9920 & Z16836 & $3380781-3380974$ & & \\
D4S2957 & 73817 & Z53093 & $3833487-3833597$ & 0.6 & \\
D4S431 & 14923 & Z17175 & $6415645-6415795$ & & \\
\hline
\end{tabular}

advisable to determine beforehand as to which markers are informative for the particular family under study. A pedigree with the result of the haplotype analysis should be included in the report.

\section{CONCLUDING REMARKS}

These guidelines are based on the knowledge acquired from peerreviewed and published data. The authors received additional information from several sources, which could not be included in the guidelines as it was unpublished. We encourage the diagnostic and scientific community to publish instructive cases or data sets to expand our knowledge of HD and its diagnosis. This will lead to a continuous improvement of our diagnostic services. These guidelines can only provide a snapshot of current knowledge at the time of publication. Readers are advised to keep up with the literature.

\section{ACKNOWLEDGEMENTS}

We thank the following people for carefully reading the manuscript and providing extremely useful comments and additional information: $\mathrm{M}$ Bost, D Cockburn, G Norbury, O O’Brien, OW Quarrell, J Sequeiros, L Snell, J Warner and C Yearwood. These guidelines were developed by a consensus process using the 1998 CMGS guidelines as a starting point. We acknowledge the contribution of Dr David Rubinsztein to the 1998 CMGS guidelines. This work was partially supported by the European Molecular Genetics Quality Network (EMQN) and by EuroGentest (EuroGentest2 Coordination Action 2011 - EU Contract no.: FP7 - HEALTH-F4-2010-261469). These draft guidelines were fully updated and published for consultation by EMQN and CMGS.

\section{INTERNET RESOURCES}

EMQN website www.emqn.org: GeneReviews funded by NIH. Developed at the University of Washington, Seattle, USA http://www.ncbi.nlm.nih.gov/ bookshelf/br.fgci?book=gene\&part=huntington: NCBI RefSeq Gene project

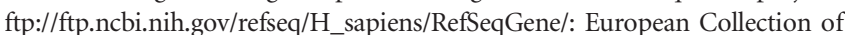
Cell Cultures http://www.ecacc.org.uk/ Cell line number CM0034, ECACC ref. no: 95090133. European Collection of Cell Cultures, 2010.: CDC Centers for Disease Control and prevention Genetic testing reference materials program $<$ link to GeT-RMs > : Coriell Cell and DNA repository http://ccr.coriell. org/sections/collections/: Clinical Molecular Genetics Society Reporting Guidelines: http://www.cmgs.org/BPGs/Reporting\%20guidelines\% 20Sept $\% 202011 \% 20$ APPROVED.pdf

1 Bates GHPJL: Huntington's disease. 3 edn, Oxford, UK: Oxford University Press, 2002.

2 Pagon RA, Tarczy-Hornoch P, Baskin PK et al: GeneTests-GeneClinics: genetic testing information for a growing audience. Hum Mutat 2002; 19: 501-509.

3 Ross CA, Tabrizi SJ: Huntington's disease: from molecular pathogenesis to clinical treatment. Lancet Neurol 2011; 10: 83-98.

4 Alonso ME, Yescas P, Rasmussen A et al: Homozygosity in Huntington's disease: new ethical dilemma caused by molecular diagnosis. Clin Genet 2002; 61: 437-442.

5 Pruitt KD, Tatusova T, Maglott DR: NCBI Reference Sequence project: update and current status. Nucleic Acids Res 2003; 31: 34-37.

6 Lee J-M, Ramos EM, Lee J-H et al: CAG repeat expansion in Huntington disease determines age at onset in a fully dominant fashion. Neurology 2012; 78: 690-695.

7 Langbehn DR, Hayden MR, Paulsen JS: CAG repeat length and the age at onset in Huntington disease (HD): a review and validation study of statistical approaches. Am J Med Genet Part B 2010; 153B: 397-408.

8 De Rooij KE, De Koning Gans PA, Skraastad MI et al: Dynamic mutation in Dutch Huntington's disease patients: increased paternal repeat instability extending to within the normal size range. J Med Genet 1993; 30: 996-1002.

9 Rubinsztein DC, Leggo J, Coles $\mathrm{R}$ et al: Phenotypic characterization of individuals with 30-40 CAG repeats in the Huntington disease (HD) gene reveals HD cases with 36 repeats and apparently normal elderly individuals with 36-39 repeats. Am J Hum Genet 1996; 59: 16-22.

10 Brinkman RR, Mezei MM, Theilmann J et al: The likelihood of being affected with Huntington disease by a particular age, for a specific CAG size. Am J Hum Genet 1997; 60: 1202-1210. 
11 Kenney C, Powell S, Jankovic J: Autopsy-proven Huntington's disease with trinucleotide repeats. Mov Disord 2007; 22: 127-130.

12 Groen JL, de Bie RMA, Foncke EMJ, Roos RAC, Leenders KL, Tijssen MAJ: Late-onset Huntington disease with intermediate CAG repeats: true or false? J Neurol Neurosurg Psychiatry 2010; 81: 228-230.

13 Andrich J, Arning L, Wieczorek S, Kraus PH, Gold R, Saft C: Huntington's disease as caused by 34 CAG repeats. Mov Disord 2008; 23: 879-881.

14 Herishanu YO, Parvari R, Pollack Y et al: Huntington disease in subjects from an Israel Karaite community carrying alleles of intermediate and expanded CAG repeats in the HTT gene: Huntington disease or phenocopy? J Neurol Sci 2009; 277: 143-146.

15 McNeil SM, Novelletto A, Srinidhi J et al: Reduced penetrance of the Huntington's disease mutation. Hum Mol Genet 1997; 6: 775-779.

16 Quarrell OW, Rigby AS, Barron L et al: Reduced penetrance alleles for Huntington's disease: a multi centre direct observational study. J Med Genet 2007; 44: e68.

17 Semaka A, Creighton S, Warby S, Hayden MR: Predictive testing for Huntington disease: interpretation and significance of intermediate alleles. Clin Genet 2006; 70: 283-294.

18 Sequeiros J, Ramos EM, Cerqueira J et al: Large normal and reduced penetrance alleles in Huntington disease: instability in families and frequency at the laboratory, at the clinic and in the population. Clin Genet 2010; 78: 381-387.

19 Hendricks AE, Latourelle JC, Lunetta KL et al: Estimating the probability of de novo $\mathrm{HD}$ cases from transmissions of expanded penetrant CAG alleles in the Huntington disease gene from male carriers of high normal alleles (27-35 CAG). Am J Med Genet A 2009; 149A: 1375-1381.

20 Goldberg YP, McMurray CT, Zeisler J et al: Increased instability of intermediate alleles 434 in families with sporadic Huntington disease compared to similar sized intermediate alleles in the general population. Hum Mol Genet 1995; 4: 1911-1918.

21 Myers RH, MacDonald ME, Koroshetz WJ et al: De novo expansion of a (CAG)n repeat in sporadic Huntington's disease. Nat Genet 1993; 5: 168-173.

22 Van Belzen MJ, Belfroid RDM, Losekoot M, Walstra GJM, Van Langen IM: Maternal intermediate repeat expansion into the affected range in Huntington's disease. Clin Genet 2009; 76(Suppl 1): 1-127.

23 Goldberg YP, Kremer B, Andrew SE et al: Molecular analysis of new mutations for Huntington's disease: intermediate alleles and sex of origin effects. Nat Genet 1993 5: $174-179$.

24 Crawford DC, Nickerson DA: Definition and clinical importance of haplotypes. Annu Rev Med 2005; 56: 303-320.

25 International Huntington Association (IHA) and the World Federation of Neurology (WFN) Research Group on Huntington's Chorea. Guidelines for the molecular genetics predictive test in Huntington's disease. Neurology 1994; 44: 1533-1536.

26 Macleod R, Tibben A, Frontali $M$ et al: Editorial committee working group, Genetic testing Counselling' of the European Huntington Disease Network. Recommendations for the predictive genetic test in Huntington's disease. Clin Genet 2012 'Accepetd Article' doi:10.1111/j.1399-0004.2012.01900.x.

27 Rij van MC, Rademaekers De M, Moutou C et al: Preimplantation Genetic diagnosis (PGD) for Huntington's disease: the experience of three European centres. Eur J Hum Genet 2012; 20: 368-375.

28 Warner JP, Barron LH, Brock DJ: A new polymerase chain reaction (PCR) assay for the trinucleotide repeat that is unstable and expanded on Huntington's disease chromosomes. Mol Cell Probes 1993; 7: 235-239.

29 Andrew SE, Goldberg YP, Theilmann J et al: ACCG repeat polymorphism adjacent to the $C A G$ repeat in the Huntington disease gene: implications for diagnostic accuracy and predictive testing. Hum Mol Genet 1994; 3: 65-67.
30 Quarerell OW, Handley O, O'Donovan $\mathrm{K}$ et al: Discrepancies in reporting the CAG repeat lengths for Huntington's disease. Eur J Hum Genet 2012; 20: 20-26.

31 Palomaki GE, Richards CS: Assessing the analytic validity of molecular testing for Huntington disease using data from an external proficiency testing survey. Genet Med 2012; 14: 69-75.

32 Kalman L, Johnson MA, Beck J et al: Development of genomic reference materials for Huntington disease genetic testing. Genet Med 2007; 9: 719-723.

33 The Huntington's Disease Collaborative Research Group. A novel gene containing a trinucleotide repeat that is expanded and unstable on Huntington's disease hromosomes. Cell 1993; 72: 971-983.

34 OECD (Organization for Economic co-operation and development) guidelines for quality assurance in molecular genetic testing 2007 copy right OECD.

35 Maat-Schieman M, Roos R, Losekoot $\mathrm{M}$ et al: Neuronal intranuclear and neuropil inclusions for pathological assessment of Huntington's disease. Brain Pathol 2007, 17: 31-37.

36 Warner JP, Barron LH, Goudie D et al: A general method for the detection of large CAG repeat expansions by fluorescent PCR J Med Genet 1996: 33. 1022-1026.

37 Gellera C, Meoni C, Castellotti B et al: Errors in Huntington disease diagnostic test caused by trinucleotide deletion in the IT15 gene. Am J Hum Genet 1996; 59: 475-477.

38 Margolis RL, Stine OC, Callahan C et al: Two novel single-base-pair substitutions adjacent to the CAG repeat in the Huntington disease gene (IT15): implications for diagnostic testing. Am J Hum Genet 1999; 64: 323-326.

39 Warner JP, Barron LH, Brock DJ: A new polymerase chain reaction (PCR) assay for the trinucleotide repeat that is unstable and expanded on Huntington's disease chromosomes. Mol Cell Probes 1993; 7: 235-239.

40 Andrew SE, Goldberg YP, Theilmann J, Zeisler J, Hayden MR: A CCG repeat polymorphism adjacent to the CAG repeat in the Huntington disease gene: implications for diagnostic accuracy and predictive testing. Hum Mol Genet 1994; 3: 65-67.

41 Cross G, Pitt T, Sharif A, Bates G, Lehrach H: False-negative results for Huntington's disease mutation. The Lancet 1994; 343: 1232.

42 Yu S, Fimmel A, Fung D, Trent RJ: Polymorphisms in the CAG repeat-a source of error in Huntington disease DNA testing. Clin Genet 2000; 58: 469-472.

43 Williams LC, Hegde MR, Nagappan R et al: Null alleles at the Huntington disease locus: implications for diagnostics and CAG repeat instability. Genet Test 2000; 4: 55-60.

44 Potter NT, Spector EB, Prior TW: Technical standards and guidelines for Huntington disease testing. Genet Med 2004; 6: 61-65.

45 Langbehn DR, Brinkman RR, Falush D et al: A new model for prediction of the age of onset and penetrance for Huntington's disease based on CAG length. Clin Genet 2004; 65: 267-277.

46 Tagle DA, Blanchard-McQuate KL, Collins FS: Dinucleotide repeat polymorphism in the Huntington's disease region at the D4S43 locus. Hum Mol Genet 1992; 1: 215.

47 Taylor SA, Barnes GT, MacDonald ME, Gusella JF: A dinucleotide repeat polymorphism at the D4S127 locus. Hum Mol Genet 1992; 1: 142.

This work is licensed under the Creative Commons Attribution-NonCommercial-No Derivative Works 3.0 Unported License. To view a copy of this license, visit http:// creativecommons.org/licenses/by-nc-nd/3.0/ 